\title{
Comprehensive Analysis of Common Different Gene Expression Signatures in the Neutrophils of Sepsis
}

\author{
Zhaojun Liu, ${ }^{1}$ Yang Chen, ${ }^{1}$ Tingting Pan, ${ }^{1}$ Jialin Liu, ${ }^{1}$ Rui Tian, ${ }^{1}$ Shaoqiong Sun, ${ }^{1}$ \\ Hongping Qu $\left(\mathbb{B},{ }^{1}\right.$ and Erzhen Chen $\mathbb{1}^{2}{ }^{2}$ \\ ${ }^{1}$ Department of Critical Care Medicine, Ruijin Hospital, Shanghai Jiao Tong University School of Medicine, 197 Ruijin Er Road, \\ Shanghai 200025, China \\ ${ }^{2}$ Department of Emergency, Ruijin Hospital, Shanghai Jiao Tong University School of Medicine, 197 Ruijin Er Road, \\ Shanghai 200025, China
}

Correspondence should be addressed to Hongping Qu; hongpingqu0412@hotmail.com and Erzhen Chen; rjchenerzhen@163.com Received 9 November 2020; Revised 16 March 2021; Accepted 31 March 2021; Published 19 April 2021

Academic Editor: Shibiao Wan

Copyright (c) 2021 Zhaojun Liu et al. This is an open access article distributed under the Creative Commons Attribution License, which permits unrestricted use, distribution, and reproduction in any medium, provided the original work is properly cited.

The central component of sepsis pathogenesis is inflammatory disorder, which is related to dysfunction of the immune system. However, the specific molecular mechanism of sepsis has not yet been fully elucidated. The aim of our study was to identify genes that are significantly changed during sepsis development, for the identification of potential pathogenic factors. Differentially expressed genes (DEGs) were identified in 88 control and 214 septic patient samples. Gene ontology (GO) and pathway enrichment analyses were performed using David. A protein-protein interaction (PPI) network was established using STRING and Cytoscape. Further validation was performed using real-time polymerase chain reaction (RT-PCR). We identified 37 common DEGs. GO and pathway enrichment indicated that enzymes and transcription factors accounted for a large proportion of DEGs; immune system and inflammation signaling demonstrated the most significant changes. Furthermore, eight hub genes were identified via PPI analysis. Interestingly, four of the top five upregulated and all downregulated DEGs were involved in immune and inflammation signaling. In addition, the most intensive hub gene $A K T 1$ and the top DEGs in human clinical samples were validated using RT-PCR. This study explored the possible molecular mechanisms underpinning the inflammatory, immune, and PI3K/AKT pathways related to sepsis development.

\section{Introduction}

Sepsis is defined as life-threatening organ dysfunction triggered by a dysregulated host response to infection $[1,2]$. Approximately 30 million people worldwide suffer from sepsis each year, which has a fatality rate of $20 \%-50 \%$. Sepsis become one of the most frequent causes of mortality in intensive care units $[3,4]$. Despite numerous advances in both fundamental and clinical research, the mortality rate for sepsis remains high [5]. Excessive inflammation leads to multiple organ failure, which is the main cause of mortality in early sepsis $[6,7]$. Diagnosing the condition early may facilitate appropriate treatment, thereby improving patient out- comes. As such, urgent identification of novel sepsis-related biomarkers is vital for its early diagnosis and monitoring and to implement relevant therapeutic interventions.

Neutrophils are the first to arrive at sites of infection or injury, where they play a vital role in the acute phase of inflammation and the innate immune response [8]. Neutrophils can activate various signaling pathways and release inflammatory mediators that amplify the inflammatory response, eventually causing multiple system organ failure [9]. Moreover, studies have shown that neutrophils play important roles in infection control during sepsis and that their biological activity is impaired during this time, leading to dysregulated immune responses [10]. Neutrophil 
activation markers may be potential biomarkers for the diagnosis and prognosis of sepsis, with many studies showing that their regulation is a key part of treatment $[11,12]$. Relevant mechanisms have been investigated, and clinical studies conducted, to find ways through which to block neutrophil dysfunction in patients with sepsis, which is a vital undertaking for the prevention and control of sepsis.

With recent advancements in genomics, transcriptional signature analysis has offered powerful insights into many disease $[13,14]$, including sepsis. Although hundreds of differentially expressed genes (DEGs) in sepsis were identified in several recently performed microarray profiling studies [15-17], few studies focus on neutrophils in sepsis. In addition, human genomic research on sepsis is limited by insufficient availability of clinical samples from a single cohort study.

In order to identify the key and promising genes or pathways associated with neutrophils in sepsis, we comprehensively reanalyzed a larger sample size (five previously collected microarrays) from the gene expression omnibus (GEO) database (https://www.ncbi.nlm.nih.gov/geo/). Common DEGs were identified and further analyzed using gene ontology (GO) annotation, pathway enrichment, and protein-protein interaction (PPI) analyses. We found that inflammation and immune response may be extremely important in the process of sepsis. These genes shed light on sepsis development, potentially providing future treatment targets.

\section{Materials and Methods}

2.1. Gene Expression Dataset Collection and Extraction. The National Center for Biotechnology Information (NCBI) GEO database is a public database supporting highthroughput gene expression data (http://www.ncbi.nlm.nih .gov/geo/). We searched GEO for relevant studies using the key words "sepsis," "homo sapiens," and "neutrophils." A dataset was included in our study if it met the following selection criteria: (1) it comprised of gene expression profiles and (2) investigated neutrophils from human whole blood; five data profiles were included: GSE49755, GSE49756, GSE49757, GSE6535, and GSE5772 [18-20]. We excluded animal studies and samples with obvious age differences (ages $<18$ and $>80$ years).

2.2. DEG Screening. DEGs were identified using GEO2R with default parameters (http://www.ncbi.nlm.nih.gov/geo/geo2r/ ). NCBI-generated annotations were used to display the DEG list. Only genes with a nominal $p$ value $<0.05$ were considered as DEGs. Common DEGs among the five GSE datasets were screened using $\mathrm{R}$ programming language. To identify overlapping genes between both groups, we used the VennDiagram function in $\mathrm{R}$.

\subsection{Functional and Pathway Enrichment Analysis. Common} genes in any four datasets were collected to gain insight into their biological functions using GO and pathway enrichment analysis (Kyoto Encyclopedia of Genes and Genomes, KEGG). DAVID is a group of online tools that provide func- tional annotations for understanding biological meaning behind a large list of genes (https://david.ncifcrf.gov/) [21, 22]. We performed $\mathrm{GO}$ and pathway enrichment analysis using DAVID, with $p<0.05$ considered statistically significant.

2.4. PPI Network Analysis. The online STRING 10.5 database [23] (https://string-db.org/) and Cytoscape [24] were used to establish a PPI network; the cut-off criterion was a combined score $>0.4$ [23]. This PPI network, in which nodes and edges represented proteins and their interactions, respectively, was subsequently visualized in Cytoscape. Furthermore, the degree of a node was equal to the number of its linked edges. Additionally, CytohHubba in Cytoscape software was used to identify hub genes. Genes with edge degrees $>10$ were defined as hub genes in this study.

2.5. Upstream Regulators of DEGs. Upstream regulatory networks from signatures of DEGs were inferred using the web application Expression2Kinases (X2K) [25] (http://X2K .cloud). We produced inferred networks of transcription factors $(\mathrm{TF})$ and intermediate proteins predicted to control the expression of the inputted gene list, by combining TF enrichment analysis with PPI network expansion.

2.6. Sample Collection and Processing. In this study, we included patients who met the criteria for sepsis according to the definition outlined by the Surviving Sepsis campaign [2] in the intensive care unit of our hospital. Samples were acquired from peripheral vein blood of three healthy volunteers (control group) and three septic patients. Detailed clinical information was shown in Table S1. Heparinized blood was collected via venipuncture of one forearm vein under aseptic conditions, and all samples were processed within $1 \mathrm{~h}$ of collection. Following density centrifugation at $500 \times$ $\mathrm{g}$ for $30 \mathrm{~min}$, neutrophils were separated from whole blood samples using Polymorphprep (AXIS-SHIELD PoC AS, Oslo, Norway). Cell purity and viability were determined by Giemsa/Wright staining and trypan blue exclusion previously described in our published paper [26].

2.7. Ethics Statement. All patients and healthy volunteers provided informed consent before participation in the study. The study was conducted in accordance with the Declaration of Helsinki, and the protocol was approved by the Ruijin Hospital Ethics Committee, Shanghai Jiao Tong University School of Medicine, China (Reference Number: 2018179 released on 20 November 2018).

2.8. RNA Isolation and Real-Time Polymerase Chain Reaction (RT-PCR). Total RNA was extracted from neutrophils using TRIzol reagent (Invitrogen, Grand Island, NY, USA), according to the manufacturer's instructions. For cDNA synthesis, RNA ( $1 \mu \mathrm{g})$ was reverse transcribed using reverse transcriptase with random hexamers as primers (PrimeScript RTPCR Kit; Takara, Kyoto, Japan). RT-PCR was performed using a SYBR Green PCR Master Mix (Takara) and the 7500 Fast RT-PCR System (Applied Biosystems, Foster City, CA, USA). Glyceraldehyde-3-phosphate dehydrogenase (GAPDH) was used as an internal control. All data were 
analyzed using the $2^{-\Delta \Delta C T}$ (CT, cycle threshold) method, and expressed as fold changes relative to reference control samples. All primer sequences used in this study are listed in Table S2.

2.9. Statistical Analysis. RT-PCR data were displayed as the mean \pm standard error of mean (SEM) and analyzed using an unpaired Student's $t$-test. All $p$ values were two-sided, with a $p<0.05$ considered statistically significant. All experiments were repeated at least three times. All statistical analyses and figures were prepared using GraphPad Prism version 6.0 (GraphPad Software, San Diego, CA, USA).

\section{Results}

3.1. Acquisition of Gene Expression Datasets. The workflow of this study is illustrated in Figure 1. After using the GEO database to search for neutrophils in patients with sepsis, five datasets were selected. Altogether, microarrays of neutrophils from 88 and 214 control and septic samples, respectively, were collected. The basic information of these datasets, such as GEO accession number, sample source, number of cases and controls, platform, and published articles, is shown in Table 1.

3.2. Identification of DEGs. Each acquired GSE dataset was analyzed using GEO2R with default parameters. There were $3437,4755,4285,2036$, and 2648 DEGs with a $p<0.05$ in GSE49755, GSE49756, GSE49757, GSE6535, and GSE5772, respectively. $\mathrm{R}$ programming language screening identified 37 common DEGs, including $16(43.2 \%)$ and 21 (56.8\%) that were up- and downregulated, respectively (Figure 2); we provided full length DEGs tables of each dataset with gene sym$\mathrm{bol} /$ name, probe id, $\operatorname{logFC}, p$ value, adj. $p$. val, and $t$ value in Table S3-S7. The top five upregulated genes were FKBP5, SORT1, VNN1, CST7, and GADD45A, while the top five downregulated genes were $P R R 5 L, S H 2 B 3, S U L F 2$, PLEKHO1, and PTPN6. These genes are listed in descending order according to the average fold change of their confirmed biological function (Table 2). Notably, the top five regulated DEGs were involved in inflammation, immune, and metabolism signaling. These results indicate that genes related to inflammation and immune signaling have a huge impact, potentially playing crucial roles in sepsis.

3.3. Functional and Pathway Enrichment Analysis. Common DEGs identified in any four datasets were classified into the following functional categories: biological process (BP), cellular compartment (CC), and molecular function (MF), according to the GO hierarchy and a threshold significance of $p<0.05$. DEGs were enriched in 43 GO-BP terms; the top 10 most significant terms are exhibited in Figure 3, including apoptotic process, intracellular signal transduction, response to hypoxia, and the Fc-gamma receptor signaling pathway involved in phagocytosis and the inflammatory response. Furthermore, these DEGs played essential roles in 16 GO-CC terms, primarily the cytosol, ruffle, and mitochondrion. These DEGs were also observed in 9 GO-MF terms, including protein binding, lipid binding, and signal transducer activity. We further used DAVID to analyze the
Search of GEO data of sepsis, homo sapines and neutrophil GEO accession

Download microarray data (http://www.ncbi.nlm.nih.gov/geo)

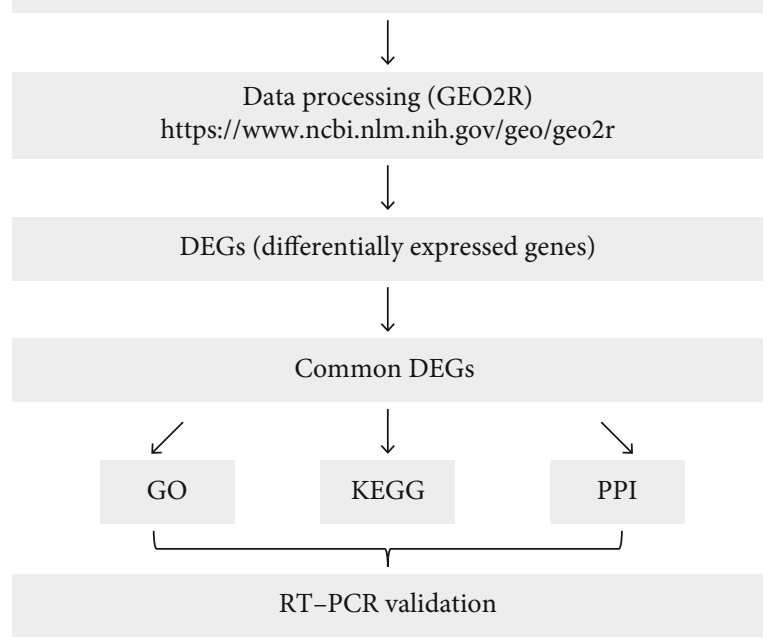

Figure 1: Overall study design.

total DEGs identified from any of the four datasets, with significantly enriched gene pathways then submitted to KEGG analysis; results are shown in Figure 4. As shown, these DEGs were enriched in 26 KEGG pathways, predominantly in autophagy regulation, Fc gamma R-mediated phagocytosis, the TNF signaling pathway, and apoptosis.

3.4. PPI Network Analysis. Using STRING, we found 175 nodes with 215 PPI relationships (Figure 5). Among these 175 nodes, eight genes were identified as hub genes with an edge degree $>10$; according to the edge degree rank, these eight genes were AKT1, GRB2, CASP8, PTGS2, SOD1, $A T G 7, M A P 2 K 1$, and TNFRSF1B. Excluding ATG7, SOD1, and $M A P 2 K 1$, all genes were downregulated; $A K T 1$ was the most intensive hub gene, interacting with 37 genes in the network. Interestingly, some hub genes could interact with multiple other hub genes. For example, $A K T 1$ could interact directly with four other hub genes, namely, ATG7, CASP8, GRB2, and MAP2K1. Together, these results suggest that eight hub genes, especially $A K T 1$, may play important roles in the development of sepsis. We further inferred networks of transcription factors (TF) and targeted differentially expressed genes using X2K (Figure 6). Transcription factors, such as RELA, CEBPB, CREB1, PPARG, RUNX1, SP11, and GATA1 have higher $k$-core values and are hubs. These are more centralized in the network and have a stronger capacity of modulating adjacent genes. Interestingly, we found that AKT1 interacts directly with other transcription factors, namely, CREB1 and GATA1. Together, these results suggest AKT1 may play important roles in the development of sepsis.

3.5. RT-PCR Validation. AKT1 and DEGs in Table 1 were randomly selected for validation. AKT1, top up- (FKBP5, SORT1, VNN1, and CST7) and downregulated (PRR5L, 
TABLE 1: Gene expression datasets for sepsis in our study.

\begin{tabular}{|c|c|c|c|c|c|c|}
\hline $\begin{array}{l}\text { GEO } \\
\text { accession }\end{array}$ & Cell type & Sepsis & Control & Range of ages (sepsis vs. control) & Platform & Published article \\
\hline GSE 49755 & $\begin{array}{l}\text { Neutrophil } \\
\text { samples }\end{array}$ & $n=24$ & $n=12$ & $61.08 \pm 3.37$ vs. $56.67 \pm 0.85$ & GPL10558 & Damien Chaussabel et al. (2014) [16] \\
\hline GSE 49756 & $\begin{array}{l}\text { Neutrophil } \\
\text { samples }\end{array}$ & $n=29$ & $n=17$ & $46.12 \pm 1.76$ vs. $65.28 \pm 2.92$ & GPL10558 & Damien Chaussabel et al. (2014) [16] \\
\hline GSE 49757 & $\begin{array}{l}\text { Neutrophil } \\
\text { samples }\end{array}$ & $n=35$ & $n=19$ & $53.54 \pm 2.46$ vs. $50.63 \pm 2.42$ & GPL10558 & Damien Chaussabel et al. (2014) [16] \\
\hline GSE 6535 & $\begin{array}{l}\text { Neutrophil } \\
\text { samples }\end{array}$ & $n=55$ & $n=17$ & I & GPL4274 & Ruby C. Y. Lin et al. (2008) [17] \\
\hline GSE 5772 & $\begin{array}{l}\text { Neutrophil } \\
\text { samples }\end{array}$ & $n=71$ & $n=23$ & I & GPL4274 & Ruby C. Y. Lin et al. (2007) [18] \\
\hline
\end{tabular}

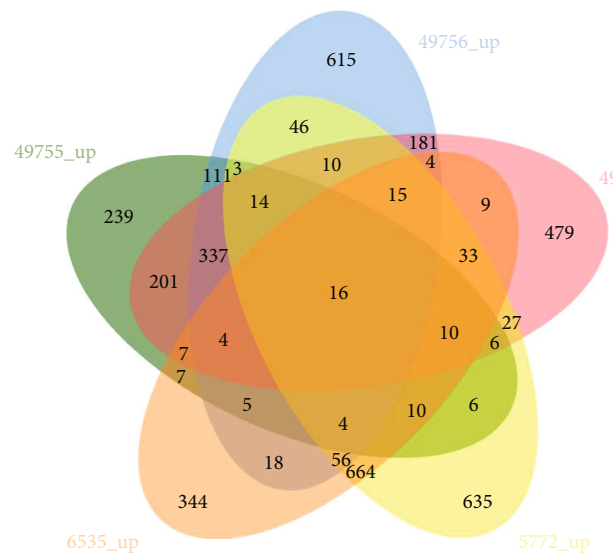

(a)

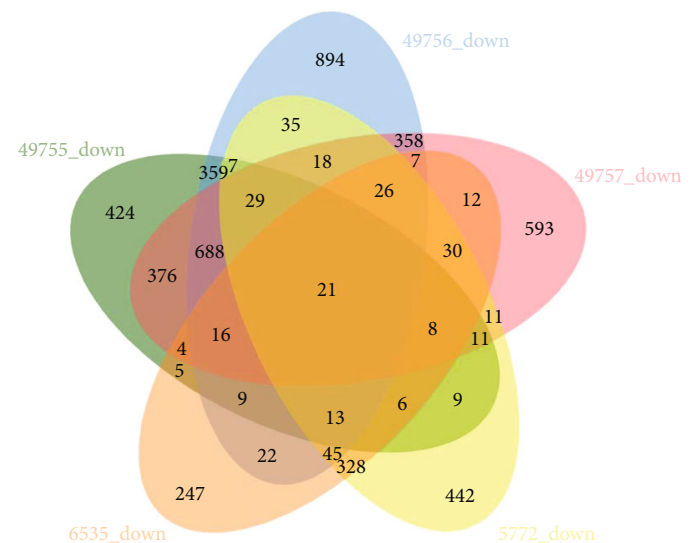

(b)

FIGURE 2: Summary of the differentially expressed genes in candidate datasets. (a) Up- and (b) downregulated genes were screened out between sepsis and controls, as shown in the Venn diagram.

TABLE 2: The top five up- and downregulated genes in sepsis and their confirmed biological function.

\begin{tabular}{lcc}
\hline Gene symbol & $\operatorname{Log~FC}_{\mathrm{A}}{ }^{1}$ & Gene title (biological function) \\
\hline FKBP5 & 1.74 & FK506 binding protein 5 (inflammation and immune system) \\
SORT1 & 1.16 & Sortilin 1 (metabolism and inflammation) \\
VNN1 & 0.84 & Vanin 1 (metabolic pathway) \\
CST7 & 0.76 & Cystatin F (immune system) \\
GADD45A & 0.75 & Growth arrest and DNA damage inducible alpha (apoptosis) \\
PRR5L & -0.95 & Proline rich protein-5 like (apoptosis) \\
SH2B3 & -0.84 & SH2B adaptor protein 3 (inflammation and immune system) \\
SULF2 & -0.75 & Sulfatase 2 (metabolism and apoptosis) \\
PLEKHO1 & -0.69 & Pleckstrin homology domain containing 01 (inflammation) \\
PTPN6 & -0.62 & Protein tyrosine phosphatase nonreceptor type 6 (inflammation)
\end{tabular}

${ }^{1} \mathrm{FC}_{\mathrm{A}}$ : average fold change of gene expression value.

SH2B3, SULF2, PLEKHO1, and PTPN6) genes in sepsis were validated using RT-PCR (as shown in Figure 7).

\section{Discussion}

Sepsis is a common cause of death in intensive care units [27]. In the early stage of sepsis, activated innate immune cells, such as neutrophils, initiate a significant increase in both innate immune and inflammatory responses to clear invading pathogens from the host. If the initial response is not properly controlled, it may result in exaggerated innate immune and inflammatory responses, leading to organ damage and increased septic mortality $[28,29]$. Consequently, a fundamental component of sepsis pathogenesis is 


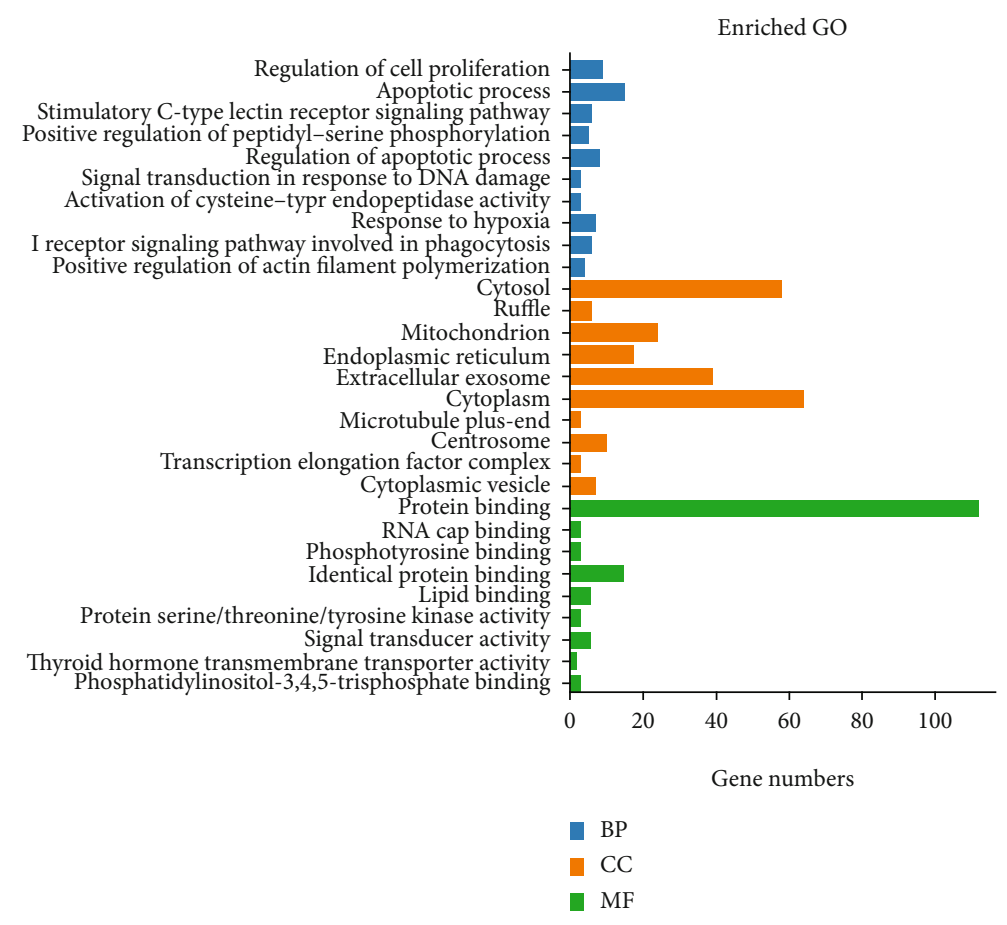

FIgure 3: Gene Ontology (GO) analysis of differentially expressed genes (DEGs) in sepsis. The top 10 GO terms enriched by DEGs in septic and normal control neutrophil samples in 3 GO categories. MF: molecular function; CC: cellular component; BP: biological process.

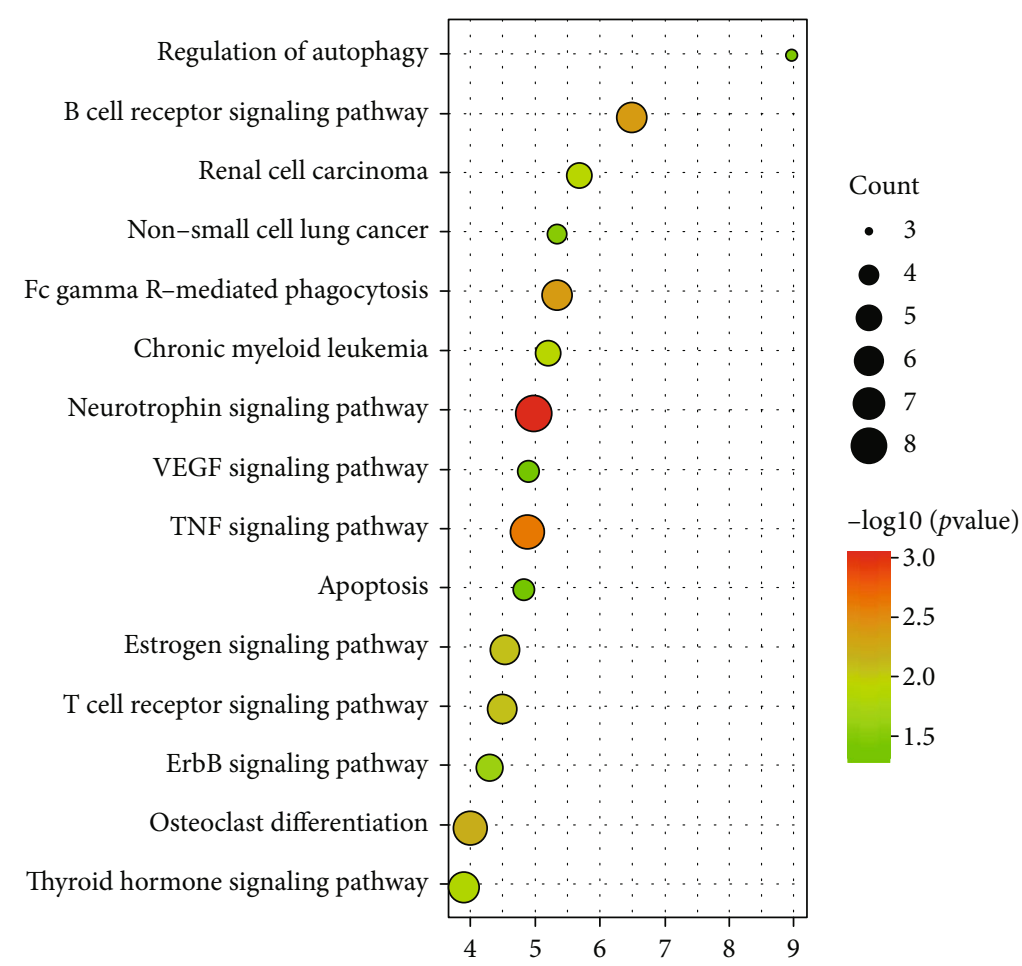

FIGURE 4: Kyoto Encyclopedia of Genes and Genomes (KEGG) pathway analysis of differentially expressed genes (DEGs) in sepsis. The top 15 KEGG pathways of DEGs in septic and normal control neutrophil samples. Colors closer to red represent higher significance.

inflammation, which is associated with immune system dysfunction [30]. High-throughput research may facilitate exploration of the critical mechanisms underpinning sepsis.
In this study, we performed a comprehensive bioinformatics analysis of neutrophil expression profiles collected from microarray studies of sepsis. Five gene expression 


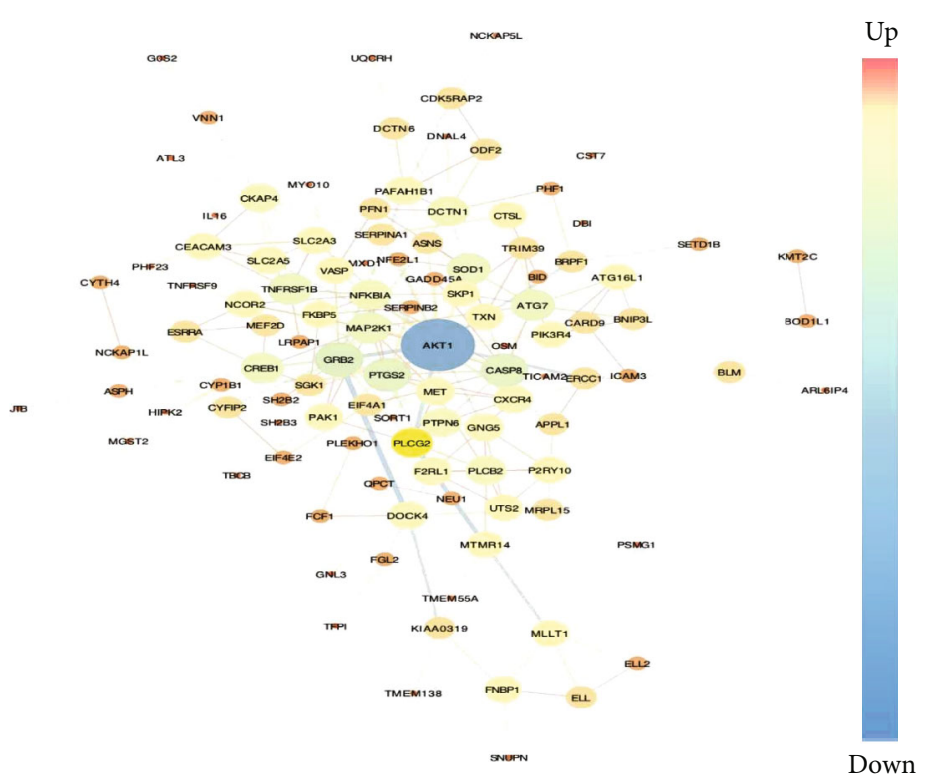

FIgURe 5: Protein-protein interaction (PPI) network complex. Functional network analysis of PPI networks based on neutrophil samples from sepsis patients. Total differentially expressed genes (DEGs) (up- and downregulated genes) were filtered into the PPI network. Red and blue nodes indicate up- and downregulated genes, respectively. Node size is proportional to edge degree. Edge color indicates significance according to the $p$ value (the brighter the color, the smaller the $p$ value).
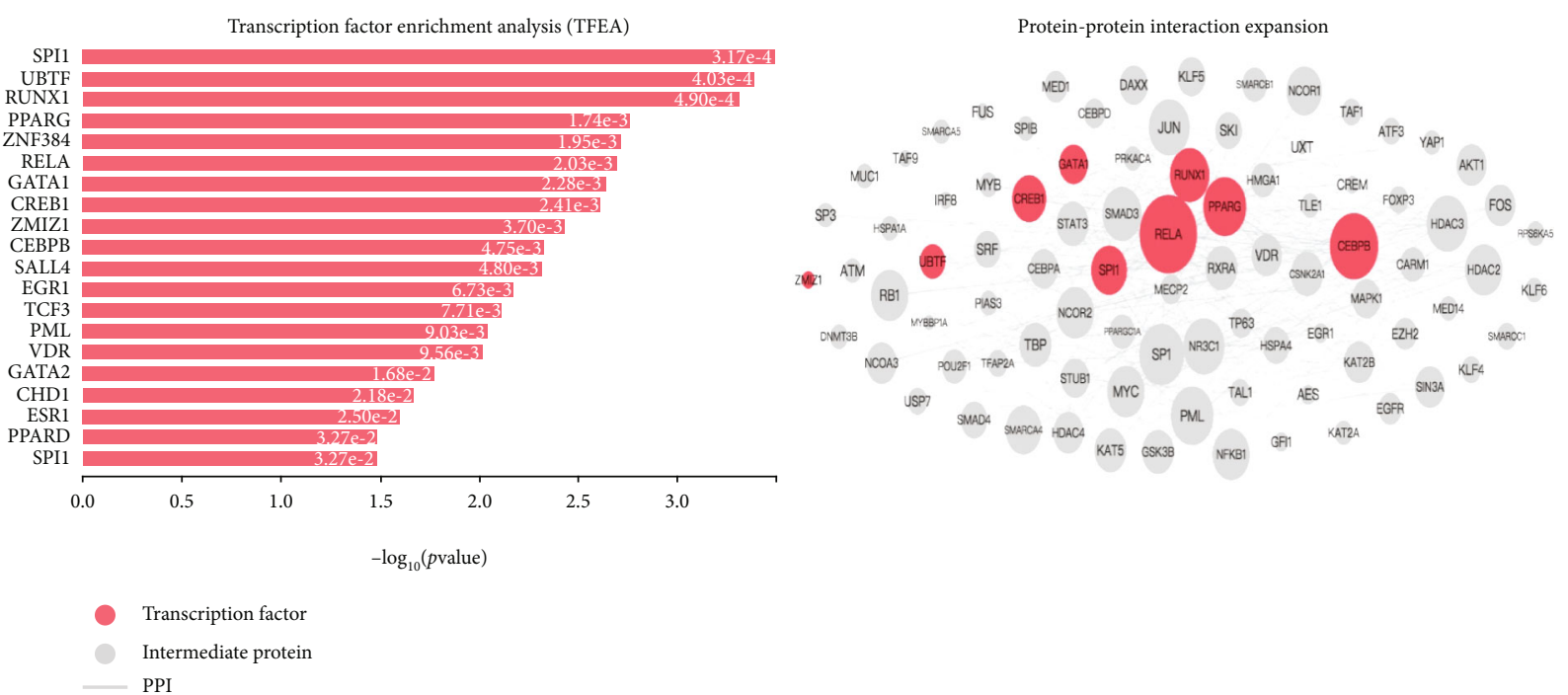

FIGURE 6: Upstream regulatory networks predicted to regulate the expression of the coregulated gene signatures in sepsis vs. the normal controls, as inferred from the Expression2Kinases (X2K) analysis. The inferred networks contain transcription factors (TFs, red nodes) and intermediate proteins (gray nodes). Gray edges indicate the interaction between two proteins (PPI). The size of nodes is relative to the level of expression degree.

profile datasets were analyzed, including 88 and 214 control and septic samples, respectively. Considering the different microarray platforms and analytical methods used in these five datasets, we reanalyzed the data using GEO2R with a uniform standard for DEGs. In total, 37 common DEGs were identified and further analyzed using functional enrichment analysis, to better understand their biological implications. Our results suggest that inflammatory response, autophagy regulation, Fc gamma R-mediated phagocytosis involved in immune responses, and the apoptotic process may be critical for the progression of sepsis. A total of 175 nodes and 215 edges were identified in the PPI network. According to PPI network analysis, AKT1 (>30) had the highest degree of interaction; all other genes were $<30$. Using clinical sepsis samples and normal healthy controls, we identified the key genes associated with inflammation in sepsis, including remarkable increases in FKBP5 and SORT1 expression, as indicated through RT-PCR studies. In addition, we identified 


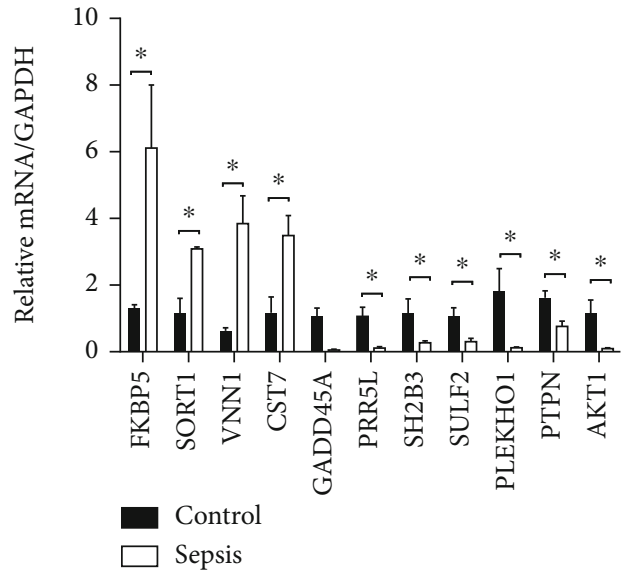

FIGURE 7: Validation of the mRNA expression level of selected genes. FKBP5, SORT1, VNN1, and CST7 were significantly upregulated in septic patients, while PRR5L, SH2B3, SULF2, PLEKHO1, PTPN6, and $A K T 1$ were significantly downregulated in septic patients $(n=3)$, compared to healthy controls $(n=3) .{ }^{*} p<0.05$.

lower expression levels of SH2B3, PLEKHO1, and PTPN6 (Figure 7).

The key finding of this study was that the inflammation signal and immune system play vital roles in the development of sepsis. The impact of hyperinflammation and immunosuppression on the development of sepsis has been studied for years $[31,32]$. There are a number of factors that contribute to immunosuppression, including apoptosis of innate immune cells, resulting in a state of immune tolerance/paralysis in which many immune cells are reprogrammed via epigenetic alterations to an unresponsive phenotype [33-35]. In our study, apoptosis, B cell receptor signaling, Fc gamma Rmediated phagocytosis, TNF, and the VEGF signaling pathway were significantly enriched in pathway analysis (Figure 4). Many aspects of the immune and inflammatory pathways were also significantly changed in our study. First, four and five of the top five up- and downregulated genes, respectively, were involved in inflammation and immune response signaling (Table 2); these corresponded to several aspects, including NF- $\kappa \mathrm{B}$ signaling-driven inflammation and immunoregulation (FKBP5) [36-38], inflammation (SORT1, SH2B3, PLEKHO1, and PTPN6) [39-42], immune response to differentiation of monocytes to macrophages (CST7) [43], and apoptosis (GADD45A, PRR5L, and SULF2) [44-46]. Second, pathway analysis indicated that half of the top ten significantly changed sepsis pathways were related to immune and inflammatory signaling (Figure 4). With regard to immune response and inflammatory signaling in sepsis, the effects of identified DEGs and pathways may be of great interest for further study.

As the highest degree gene obtained through PPI network analysis, $A K T 1$ is a key regulator of the phosphoinositide 3 kinase (PI3K)/Akt signaling cascade, which controls cell growth and survival $[47,48]$. Previous reports indicate that low molecular mass hyaluronan suppresses neutrophil apoptosis to trigger pulmonary inflammation via activating the PI3K/Akt pathway [49]. Apoptosis and phosphatidylinositol-3,4,5-trisphosphate (PIP3, a lipid second messenger formed by PI3K) binding and the TNF signaling pathway were significantly enriched in our pathway analysis. Moreover, AKT1 has been reported functionally related with sepsis. Ling et al. showed that inhibition of VNN1 can alleviate lung injury through activation of the AKT signaling pathway in septic shock [50]. Polymicrobial sepsis causes cardiac dysfunction that is linked to activation of Akt signaling pathways [51]. What is more, expression of $A K T 1$ in septic patients was significantly decreased compared to healthy controls using RT-PCR validation (Figure 7). Therefore, the PI3K/Akt pathway in neutrophils may play a proinflammatory role in patients with sepsis.

Our study identified that FKBP5 was the topmost upregulated gene in septic patients, compared to normal healthy controls. FKBP5, also as the regulatory gene of $A K T 1$, encodes for a cochaperone protein that is acutely induced by stress and which can regulate immune and basic cellular processes involved in protein folding and trafficking [37, $38,52]$. Experiments in $\mathrm{T}$ immune cells showed that higher FKBP5 promotes inflammation by strengthening the interactions of NF- $\kappa \mathrm{B}$ regulatory kinases [36]. The upregulation of FKBP5 expression has been observed not only in stress exposure and glucocorticoid stimulation but also in melanoma, viral infection, depression, and some other diseases [5355]. However, no studies have reported the expression level and role of FKBP5 in septic neutrophils. A dysregulated inflammatory response is one of the major characteristics of sepsis. Overexpression of FKBP5, as observed in our results, may play a role in promoting inflammation signaling in sepsis.

Although our analysis utilizes high-throughput and a large sample size, there are still limitations in the present work. First, molecular docking studies of hub genes would be needed to perform for modelling the interaction between a small molecule and a protein at the atomic level associated with sepsis. Second, more clinical samples and further relevant experimental assays, including animal models, should be conducted to confirm the underlying biological roles of key genes and pathways in our analysis of sepsis.

\section{Conclusions}

In conclusion, we offer a novel and comprehensive analysis of neutrophil gene expression profiles in clinical sepsis samples. Genes involved in inflammatory, immune, and PI3K/AKT pathways were significantly changed in sepsis. Our analysis provides valuable information for future research into the molecular mechanisms underpinning sepsis, thereby offering clues for the discovery of novel therapeutic strategies.

\section{Data Availability}

The datasets supporting the conclusions of this article are within the article and its additional files.

\section{Conflicts of Interest}

The authors declare that they have no conflict of interest. 


\section{Authors' Contributions}

Hongping Qu and Erzhen Chen are responsible for the conceptualization; Zhaojun Liu for the data curation; Tingting Pan and Zhaojun Liu for the methodology; Yang Chen for the software; Rui Tian and Shaoqiong Sun for the validation; Tingting Pan for the visualization; Zhaojun Liu and Yang Chen for the writing-original draft; and Jialin Liu, Hongping Qu, and Erzhen Chen for the review and editing. All authors read and approved the final manuscript. Zhaojun Liu and Yang Chen contributed equally to this work.

\section{Acknowledgments}

We thank the patients and healthy volunteers who participated in our study. This work was funded by the National Natural Science Foundation of China (No. 81772040) and Project of Shanghai Municipal Commission of Health and Family Planning (No. ZY(2018-2020)-FWTX-1108).

\section{Supplementary Materials}

Supplementary 1.

Supplementary 2.

Supplementary 3.

Supplementary 4.

Supplementary 5.

Supplementary 6.

Supplementary 7.

\section{References}

[1] C. W. Seymour, V. X. Liu, T. J. Iwashyna et al., "Assessment of clinical criteria for sepsis: for the third international consensus definitions for sepsis and septic shock (sepsis-3)," JAMA, vol. 315, no. 8, pp. 762-774, 2016.

[2] M. Singer, C. S. Deutschman, C. W. Seymour et al., "The third international consensus definitions for sepsis and septic shock (sepsis-3)," JAMA, vol. 315, no. 8, pp. 801-810, 2016.

[3] C. Fleischmann, A. Scherag, N. K. Adhikari et al., "Assessment of global incidence and mortality of hospital-treated sepsis. Current estimates and limitations," American Journal of Respiratory and Critical Care Medicine, vol. 193, no. 3, pp. 259-272, 2016.

[4] C. S. Deutschman and K. J. Tracey, "Sepsis: current dogma and new perspectives,” Immunity, vol. 40, no. 4, pp. 463-475, 2014.

[5] R. Herrán-Monge, A. Muriel-Bombín, M. M. García-García et al., "Epidemiology and changes in mortality of sepsis after the implementation of surviving sepsis campaign guidelines," Journal of Intensive Care Medicine, vol. 34, no. 9, pp. 740750, 2019.

[6] R. S. Hotchkiss, G. Monneret, and D. Payen, "Sepsis-induced immunosuppression: from cellular dysfunctions to immunotherapy," Nature Reviews Immunology, vol. 13, no. 12, pp. 862-874, 2013.

[7] R. Ding, Y. Meng, and X. Ma, "The central role of the inflammatory response in understanding the heterogeneity of sepsis-
3," BioMed Research International, vol. 2018, Article ID 5086516, 10 pages, 2018.

[8] C. Summers, S. M. Rankin, A. M. Condliffe, N. Singh, A. M. Peters, and E. R. Chilvers, "Neutrophil kinetics in health and disease," Trends in Immunology, vol. 31, no. 8, pp. 318-324, 2010.

[9] X. F. Shen, K. Cao, J. P. Jiang, W. X. Guan, and J. F. du, "Neutrophil dysregulation during sepsis: an overview and update," Journal of Cellular and Molecular Medicine, vol. 21, no. 9, pp. 1687-1697, 2017.

[10] G. F. Zarbato, M. P. de Souza Goldim, A. D. Giustina et al., "Dimethyl fumarate limits neuroinflammation and oxidative stress and improves cognitive impairment after polymicrobial sepsis," Neurotoxicity Research, vol. 34, no. 3, pp. 418-430, 2018.

[11] J. Chen, B. Wang, J. Lai et al., "Trimetazidine attenuates cardiac dysfunction in endotoxemia and sepsis by promoting neutrophil migration," Frontiers in Immunology, vol. 9, p. 2015, 2018.

[12] E. Sapey, J. M. Patel, H. Greenwood et al., "Simvastatin improves neutrophil function and clinical outcomes in pneumonia. A pilot randomized controlled clinical trial," American Journal of Respiratory and Critical Care Medicine, vol. 200, no. 10, pp. 1282-1293, 2019.

[13] Y. Li, Q. Jiang, Z. Ding et al., "Identification of a common different gene expression signature in ischemic cardiomyopathy," Genes, vol. 9, no. 1, p. 56, 2018.

[14] C. D. Savci-Heijink, H. Halfwerk, J. Koster, H. M. Horlings, and M. J. van de Vijver, "A specific gene expression signature for visceral organ metastasis in breast cancer," BMC Cancer, vol. 19, no. 1, p. 333, 2019.

[15] Y. Qi, X. Chen, N. Wu, C. Ma, X. Cui, and Z. Liu, "Identification of risk factors for sepsis-associated mortality by gene expression profiling analysis," Molecular Medicine Reports, vol. 17, no. 4, pp. 5350-5355, 2018.

[16] H. Chen, Y. Li, T. Li et al., "Identification of potential transcriptional biomarkers differently expressed in both S. aureusand E. coli-induced sepsis via integrated analysis," BioMed Research International, vol. 2019, Article ID 2487921, 11 pages, 2019.

[17] S. Ahmad, P. Singh, A. Sharma et al., "Transcriptome metaanalysis deciphers a dysregulation in immune responseassociated gene signatures during sepsis," Genes, vol. 10, no. 12, p. 1005, 2019.

[18] P. Khaenam, D. Rinchai, M. C. Altman et al., "A transcriptomic reporter assay employing neutrophils to measure immunogenic activity of septic patients' plasma," Journal of Translational Medicine, vol. 12, no. 1, p. 65, 2014.

[19] B. M. Tang, A. S. McLean, I. W. Dawes, S. J. Huang, M. J. Cowley, and R. C. Y. Lin, "Gene-expression profiling of gram-positive and gram-negative sepsis in critically ill patients," Critical Care Medicine, vol. 36, no. 4, pp. 1125$1128,2008$.

[20] B. M. Tang, A. S. McLean, I. W. Dawes, S. J. Huang, and R. C. Y. Lin, "The use of gene-expression profiling to identify candidate genes in human sepsis," American Journal of Respiratory and Critical Care Medicine, vol. 176, no. 7, pp. 676-684, 2007.

[21] D. W. Huang, B. T. Sherman, and R. A. Lempicki, "Systematic and integrative analysis of large gene lists using DAVID bioinformatics resources," Nature Protocols, vol. 4, no. 1, pp. 44-57, 2009. 
[22] D. W. Huang, B. T. Sherman, and R. A. Lempicki, "Bioinformatics enrichment tools: paths toward the comprehensive functional analysis of large gene lists," Nucleic Acids Research, vol. 37, no. 1, pp. 1-13, 2009.

[23] D. Szklarczyk, J. H. Morris, H. Cook et al., "The STRING database in 2017: quality-controlled protein-protein association networks, made broadly accessible," Nucleic Acids Research, vol. 45, no. D1, pp. D362-D368, 2017.

[24] N. T. Doncheva, Y. Assenov, F. S. Domingues, and M. Albrecht, "Topological analysis and interactive visualization of biological networks and protein structures," Nature Protocols, vol. 7, no. 4, pp. 670-685, 2012.

[25] D. J. B. Clarke, M. V. Kuleshov, B. M. Schilder et al., "eXpression2Kinases (X2K) Web: linking expression signatures to upstream cell signaling networks," Nucleic Acids Research, vol. 46, no. W1, pp. W171-W179, 2018.

[26] Y. Chen, Z. Liu, T. Pan et al., "JMJD3 is involved in neutrophil membrane proteinase 3 overexpression during the hyperinflammatory response in early sepsis," International Immunopharmacology, vol. 59, pp. 40-46, 2018.

[27] for the China Critical Care Clinical Trials Group (CCCCTG), L. Weng, X. Y. Zeng et al., "Sepsis-related mortality in China: a descriptive analysis," Intensive Care Medicine, vol. 44, no. 7, pp. 1071-1080, 2018.

[28] B. G. Chousterman, F. K. Swirski, and G. F. Weber, "Cytokine storm and sepsis disease pathogenesis," Seminars in Immunopathology, vol. 39, no. 5, pp. 517-528, 2017.

[29] V. Vachharajani and C. E. McCall, "Epigenetic and metabolic programming of innate immunity in sepsis," Innate Immunity, vol. 25, no. 5, pp. 267-279, 2019.

[30] M. Cecconi, L. Evans, M. Levy, and A. Rhodes, "Sepsis and septic shock," Lancet, vol. 392, no. 10141, pp. 75-87, 2018.

[31] H. Yadav and R. Cartin-Ceba, "Balance between hyperinflammation and immunosuppression in sepsis," Seminars in Respiratory and Critical Care Medicine, vol. 37, no. 1, pp. 042-050, 2016.

[32] J. C. Mira, L. F. Gentile, B. J. Mathias et al., "Sepsis pathophysiology, chronic critical illness, and persistent inflammationimmunosuppression and catabolism syndrome," Critical Care Medicine, vol. 45, no. 2, pp. 253-262, 2017.

[33] C. Cao, M. Yu, and Y. Chai, "Pathological alteration and therapeutic implications of sepsis-induced immune cell apoptosis," Cell Death \& Disease, vol. 10, no. 10, p. 782, 2019.

[34] R. J. Arts, M. S. Gresnigt, L. A. Joosten, and M. G. Netea, "Cellular metabolism of myeloid cells in sepsis," Journal of Leukocyte Biology, vol. 101, no. 1, pp. 151-164, 2017.

[35] W. F. Carson, K. A. Cavassani, Y. Dou, and S. L. Kunkel, "Epigenetic regulation of immune cell functions during post-septic immunosuppression," Epigenetics, vol. 6, no. 3, pp. 273-283, 2011.

[36] A. S. Zannas, M. Jia, K. Hafner et al., "Epigenetic upregulation of FKBP5 by aging and stress contributes to NF- $\kappa \mathrm{B}$-driven inflammation and cardiovascular risk," Proceedings of the National Academy of Sciences of the United States of America, vol. 116, no. 23, pp. 11370-11379, 2019.

[37] A. C. Bustamante, A. E. Aiello, G. Guffanti, S. Galea, D. E. Wildman, and M. Uddin, "FKBP5 DNA methylation does not mediate the association between childhood maltreatment and depression symptom severity in the Detroit Neighborhood Health Study," Journal of Psychiatric Research, vol. 96, pp. 39-48, 2018.
[38] L. Tozzi, C. Farrell, L. Booij et al., "Epigenetic changes of FKBP5 as a link connecting genetic and environmental risk factors with structural and functional brain changes in major depression," Neuropsychopharmacology, vol. 43, no. 5, pp. 1138-1145, 2018.

[39] M. B. Mortensen, M. Kjolby, S. Gunnersen et al., "Targeting sortilin in immune cells reduces proinflammatory cytokines and atherosclerosis," The Journal of Clinical Investigation, vol. 124, no. 12, pp. 5317-5322, 2014.

[40] J. Devallière and B. Charreau, "The adaptor Lnk (SH2B3): an emerging regulator in vascular cells and a link between immune and inflammatory signaling," Biochemical Pharmacology, vol. 82, no. 10, pp. 1391-1402, 2011.

[41] X. He, J. Liu, C. Liang et al., "Osteoblastic PLEKHO1 contributes to joint inflammation in rheumatoid arthritis," eBioMedicine, vol. 41, pp. 538-555, 2019.

[42] M. Speir, C. J. Nowell, A. A. Chen et al., "Ptpn 6 inhibits caspase-8 and Ripk 3/Mlkl-dependent inflammation," Nature Immunology, vol. 21, no. 1, pp. 54-64, 2020.

[43] E. Dautovic, M. Perisic Nanut, A. Softic, and J. Kos, “The transcription factor C/EBP $\alpha$ controls the role of cystatin F during the differentiation of monocytes to macrophages," European Journal of Cell Biology, vol. 97, no. 7, pp. 463-473, 2018.

[44] R. Bartoszewski, M. Gebert, A. Janaszak-Jasiecka et al., "Genome-wide mRNA profiling identifies RCAN1 and GADD45A as regulators of the transitional switch from survival to apoptosis during ER stress," The FEBS Journal, vol. 287, no. 14, pp. 2923-2947, 2020.

[45] K. Thedieck, P. Polak, M. L. Kim et al., "PRAS40 and PRR5like protein are new $\mathrm{mTOR}$ interactors that regulate apoptosis," PLoS One, vol. 2, no. 11, article e1217, 2007.

[46] T. Jiang, Z. H. Chen, Z. Chen, and D. Tan, "SULF2 promotes tumorigenesis and inhibits apoptosis of cervical cancer cells through the ERK/AKT signaling pathway," Brazilian Journal of Medical and Biological Research, vol. 53, no. 2, article e8901, 2020.

[47] B. D. Manning and L. C. Cantley, "AKT/PKB signaling: navigating downstream," Cell, vol. 129, no. 7, pp. 1261-1274, 2007.

[48] H. Akrami, F. Mahmoodi, S. Havasi, and A. Sharifi, "PlGF knockdown inhibited tumor survival and migration in gastric cancer cell via PI3K/Akt and p38MAPK pathways," Cell Biochemistry and Function, vol. 34, no. 3, pp. 173-180, 2016.

[49] H. Zhao, Y. Ma, and L. Zhang, "Low-molecular-mass hyaluronan induces pulmonary inflammation by up-regulation of Mcl-1 to inhibit neutrophil apoptosis via PI3K/Akt 1 pathway," Immunology, vol. 155, no. 3, pp. 387-395, 2018.

[50] L. Ling, H. T. Lu, H. F. Wang, M. J. Shen, and H. B. Zhang, "MicroRNA-203 acts as a potent suppressor in septic shock by alleviating lung injury via inhibition of VNN1," Kidney \& Blood Pressure Research, vol. 44, no. 4, pp. 565-582, 2019.

[51] F. Fattahi, M. Kalbitz, E. A. Malan et al., "Complementinduced activation of MAPKs and Akt during sepsis: role in cardiac dysfunction," The FASEB Journal, vol. 31, no. 9, pp. 4129-4139, 2017.

[52] P. Misra, S. Liu, and X. Meng, "What DNA methylation modifications and/or genetic variations interact with childhood maltreatment in the development of depression: a systematic review," Journal of Affective Disorders, vol. 252, pp. 325-333, 2019.

[53] A. S. Zannas, T. Wiechmann, N. C. Gassen, and E. B. Binder, "Gene-stress-epigenetic regulation of FKBP5: clinical and 
translational implications," Neuropsychopharmacology, vol. 41, no. 1, pp. 261-274, 2016.

[54] W. Hao, L. Wang, and S. Li, "FKBP5 regulates RIG-I-mediated NF- $\kappa \mathrm{B}$ activation and influenza A virus infection," Viruses, vol. 12, no. 6, p. 672, 2020.

[55] S. Romano, E. Simeone, A. D'Angelillo et al., "FKBP51s signature in peripheral blood mononuclear cells of melanoma patients as a possible predictive factor for immunotherapy," Cancer Immunology, Immunotherapy, vol. 66, no. 9, pp. 1143-1151, 2017. 